\title{
A Study on the Overheating of the Power Cable Tray
}

\author{
Hyung-Joo Choi *, and Heung-Ho Lee **
}

\begin{abstract}
This paper includes the results of the electromagnetic finite element analysis with regard to overheating problem of the power cable tray due to asymmetric magnetic flux density. This phenomenon was experienced in the utility power plant, Korea. The influences of the power cable arrangements and material of the tray were analyzed to find the best solutions using the eddy current-thermal coupled analysis.
\end{abstract}

Keywords: Core loss, Asymmetric flux, Eddy-current, Thermal coupled analysis, Cable tray overheating, Finite element analysis, Flat and Trefoil type, Cable arrangement

\section{Introduction}

\subsection{Overheating of the power cable tray}

The four power cables per phase have been installed on the tray between the Auxiliary Transformer and the medium voltage $(6.9 \mathrm{kV})$ metal-clad switchgear bus as illustrated in Fig. 1.

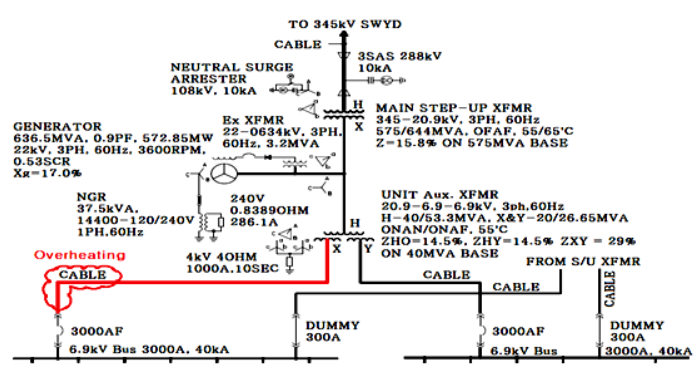

Fig. 1. Overheating location of the power cable tray
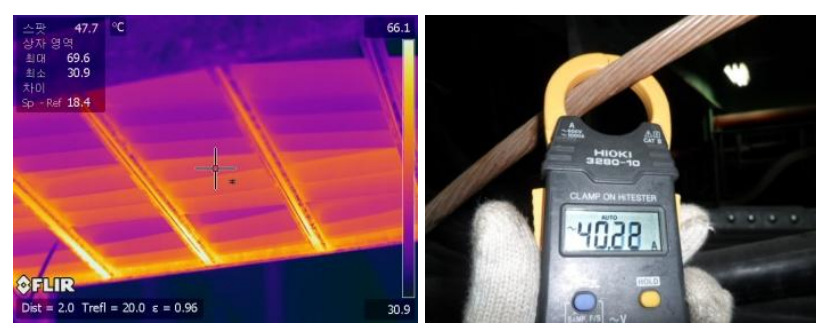

(a) Temperature on the Tray (b) Current of ground wire Fig. 2. Overheating on the tray and current of ground wire

An overheating problem up to 70 degrees Celsius on the ladder rung of tray has been identified using a thermal

\footnotetext{
* Dept. of electrical Eng. Korea Midland Power Company(KOMIPO), Korea (nimble@komipo.co.kr)

** Department of Electrical Engineering, Chung-Nam National University, Korea (leehh@cnu.ac.kr)

Received 21 October 2013; Accepted 3 November 2013
}

camera. Also, the induced current has been measured up to $270 \mathrm{~A}$ at end of the bared ground wire. Its magnitude is increased in proportional to the distance of power cables in parallel.

\subsection{Power cable arrangement}

In general, three-phase power cables on the tray should be aligned with either Flat type or Trefoil type [1]-[3].

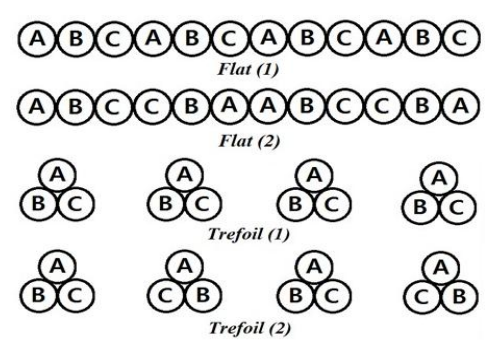

Fig. 3. Various types of power cable arrangement

All of the power cables were installed with the Trefoil type in the utility power plant. However, some power cables in overheating area were randomly aligned with the Flat type within approximately $20 \mathrm{~m}$ from the switchgear as shown in Fig. 4. So it is necessary to analyze the relationship between asymmetric magnetic flux and core loss of cable tray.

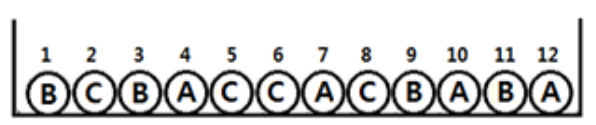

Flat (3)

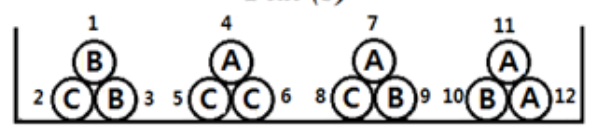

Trefoil (3)

Fig. 4. Installation of the three-phase power cables 


\section{Electromagnetic Finite Element Analysis}

\subsection{Simulation Condition}

When the magnetic materials are exposed to a timevarying field, the induced voltages from the time-varying field cause currents as known eddy currents to flow in the conductors. The currents will be concentrated near the surface of the conductors decaying rapidly past the skin depth. It is defined as the depth at which the current density decays to $1 / \mathrm{e}$ of its maximum value, as much as $86 \%$ of the current flows within the outer surface of materials equal to the skin depth. Induced currents allow magnetic fields to penetrate conductors only to a certain depth, which is approximated in equation (1).

$$
\delta=\sqrt{\frac{2}{\omega \sigma \mu_{0} \mu_{r}}}
$$

Where

$\delta:$ Standard depth of penetration $(m)$

$\omega:$ Angular frequency, which is equal to $(2 \pi f)$

$f$ : Frequency at witch source currents and voltages

$\sigma$ : Conductor's conductivity $(S / m)$

$\mu_{0}$ : Permeability of free space $\left(4 \pi \times 10^{-7} \mathrm{H} / \mathrm{m}\right)$

$\mu_{r}:$ Relative permeability

The skin depth of the iron material can be calculated 0.32 $\mathrm{mm}$ at the fundamental frequency $60 \mathrm{~Hz}$ whereas that of the aluminum as non-magnetic materials is $10.54 \mathrm{~mm}$ as shown in the following Table 1. The length and thickness of the tray were assumed to be $1.25 \mathrm{~m}, 2 \mathrm{~mm}$ respectively. The $630 \mathrm{~mm} 2$ power cable consists of the 53 stranded copper conductors but the insulation and shields of the power cables are ignored in order to avoid complex boundary conditions.

Table 1. Parameter of materials

\begin{tabular}{l|c|c|c}
\hline \multirow{2}{*}{ Section } & \multicolumn{3}{|c}{ Materials of the tray } \\
\cline { 2 - 4 } & Copper & Iron & Aluminum \\
\hline $\begin{array}{l}\text { Relative Permittivity } \\
(\mathrm{A} / \mathrm{m})\end{array}$ & 1 & $4 \mathrm{E}+03$ & 1.000021 \\
\hline $\begin{array}{l}\text { Bulk Conductivity } \\
(\text { Simens } / \mathrm{m})\end{array}$ & $5.80 \mathrm{E}+07$ & $1.03 \mathrm{E}+07$ & $3.80 \mathrm{E}+07$ \\
\hline Skin Depth $(\mathrm{mm})$ & 8.53 & 0.32 & 10.54 \\
\hline $\begin{array}{l}\text { Temperature } \\
\text { Coefficient }\left(T_{c}\right)\end{array}$ & 0.004 & 0.006 & 0.0043 \\
\hline
\end{tabular}

The Triangular-shaped meshes of the materials can be generated on the basis of the skin-depth of materials in the
Electromagnetic Finite Element Analysis, Software Maxwell V16.0, and its surface was set to a finer mesh with 2 layers as shown in Fig. 5.

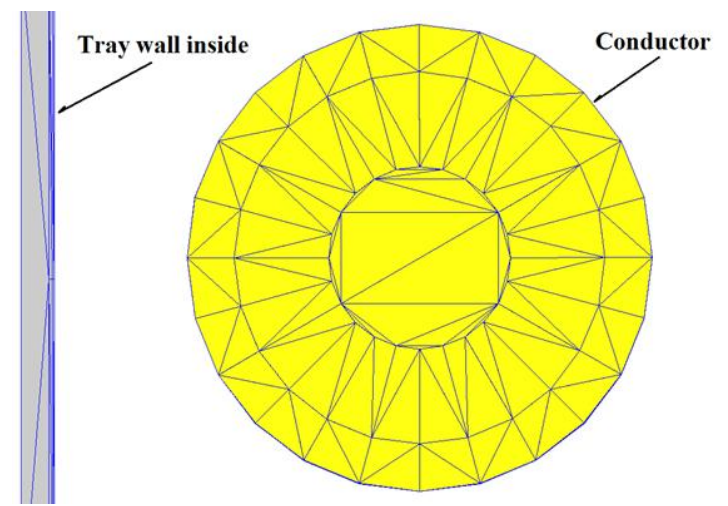

Fig. 5. Mesh status of the tray and conductor

All conductors are energized by voltage source $4.157 \mathrm{kV}$, $2 \mathrm{kA}$ per phase with $60 \mathrm{~Hz}$ (fundamental positive sequence), $180 \mathrm{~Hz}$ (zero sequence) and $300 \mathrm{~Hz}$ (negative sequence). The winding can be configured easily using an external circuit with 5\% 3rd, 3\% 5th harmonics in phase and balanced inductive loads. A uniform current density is assumed throughout the stranded conductor without computing eddy currents and displacement currents. However, the current flowing through individual conductors in the parallel group is unconstrained and its value is computed during the solution.

$$
\begin{aligned}
v_{a}= & v_{1}(\sin (\omega t))+v_{3}(\sin (3 \omega t))+v_{5}(\sin (5 \omega t)) \\
v_{b}= & v_{1}(\sin (\omega t+120))+v_{3}(\sin (3 \omega t+120)) \\
& +v_{5}(\sin (5 \omega t+120)) \\
v_{c}= & v_{1}(\sin (\omega t-120))+v_{3}(\sin (3 \omega t-120)) \\
& +v_{5}(\sin (5 \omega t-120))
\end{aligned}
$$

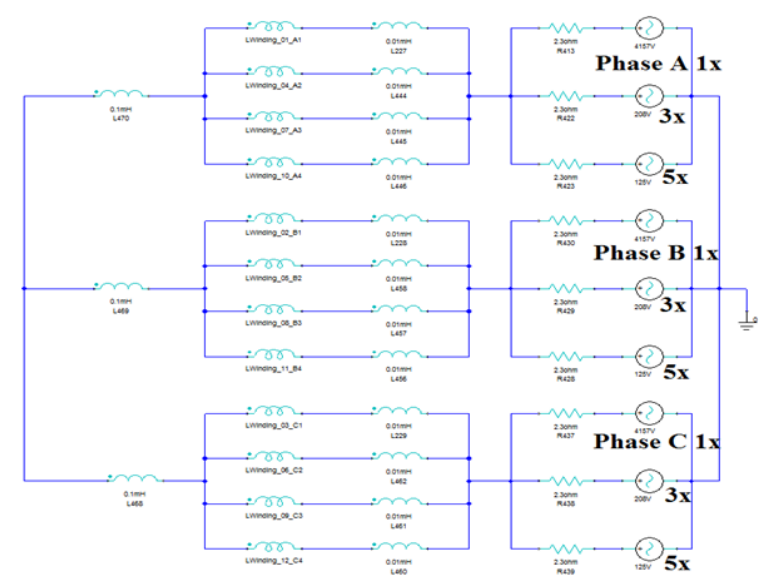

Fig. 6. External three-phase voltage source with harmonics 


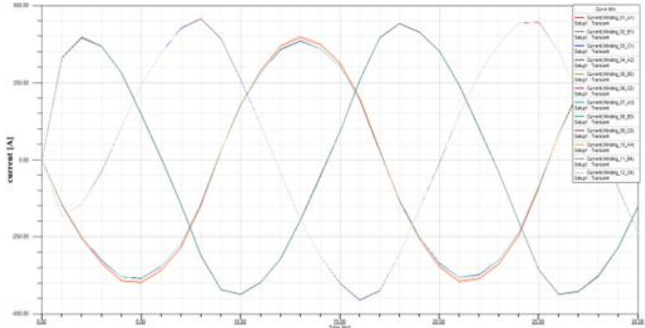

Fig. 7. Voltage waveform of the conductors

\subsection{Current distribution analysis}

An impedance matrix can represent the relationship between $\mathrm{AC}$ voltages and $\mathrm{AC}$ currents in multi-conductor system.

$$
\left[\begin{array}{c}
V_{a} \\
V_{b} \\
V_{c}
\end{array}\right]=\left[\begin{array}{lll}
Z_{a a} & Z_{a b} & Z_{a c} \\
Z_{b a} & Z_{b b} & Z_{b c} \\
Z_{c a} & Z_{c b} & Z_{c c}
\end{array}\right] \cdot\left[\begin{array}{c}
I_{a} \\
I_{b} \\
I_{c}
\end{array}\right]
$$

Where

$Z_{a a}=R_{a a}+j \omega L_{a a}:$ self-impedance of a-phase.

$Z_{a b}=R_{a b}+j \omega L_{a b}:$ mutual impedance between a-b phase.

$Z_{a c}=R_{a c}+j \omega L_{a c}:$ mutual impedance between a-c phase.

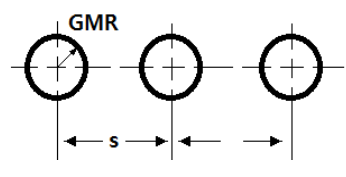

(a) Flat type

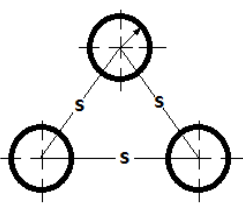

(b) Trefoil type
Fig. 8. Comparison of impedance between the align types

The inductance to be given to each phase can be computed by means of equation (4).

$$
L=0.2 \ln \left(\frac{K \cdot s}{G M R}\right) \quad[\mathrm{mH} / \mathrm{km}]
$$

where

$$
s \text { : axial spacing }[\mathrm{mm}]
$$

$K: 1$ for trefoil arrangement, 1.26 for flat laying, It can be confirmed by means of the Geometrical Mean Distance among conductor centers, $G M D=\sqrt[3]{S_{12} \cdot S_{23} \cdot S_{13}}$

GMR: Geometrical mean radius of the phase conductor

Total phase current $I_{A}, I_{B}$ and $I_{C}$ can be expressed in relation to loop currents $I_{a i}, I_{b i}$ and $I_{c i}$ in their corresponding phases.

$$
I_{A}=\sum_{i=1}^{n} Z_{a i}^{-1} V_{a i}, I_{B}=\sum_{i=1}^{n} Z_{b i}^{-1} V_{b i}, I_{C}=\sum_{i=1}^{n} Z_{c i}^{-1} V_{c i}
$$

It is found that the currents of conductors are distributed evenly in the Flat (2) type and the Trefoil (2) types under symmetrical excitation currents as shown in Fig 9. In case the power cables are arranged in random such as the Flat (3) type and the Trefoil (3) type, the current flowing through the conductors is concentrated on some conductors.

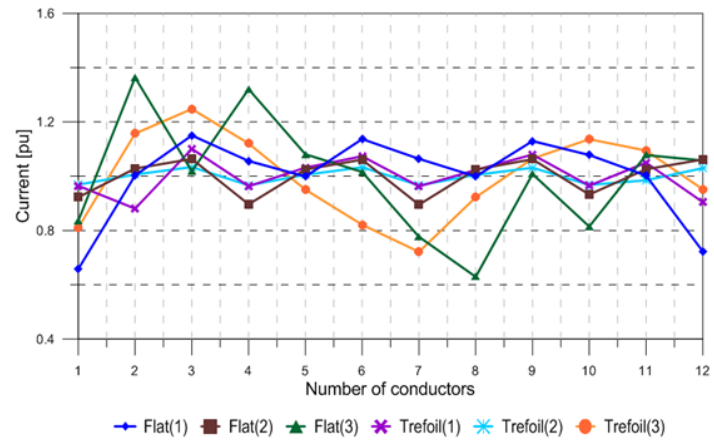

Fig. 9. Current distribution in Flat cable arrangement

\subsection{Losses analysis}

Both the solid loss and the stranded loss of an object represent the resistive loss in a volume and it is calculated integrating current density $(J)$ over a volume using the equation (6).

$$
P_{r}=\frac{1}{\sigma} \int_{v o l} J^{2} d v(W)
$$

The stranded loss is always associated with conduction current distribution in conductors which are not perfect. Thus the resistivity of conductors is responsible for the power loss when current flows in such conductors. As depicted in figure bellow, the current unbalance significantly increases in the Flat (3) type and Trefoil (3) type.

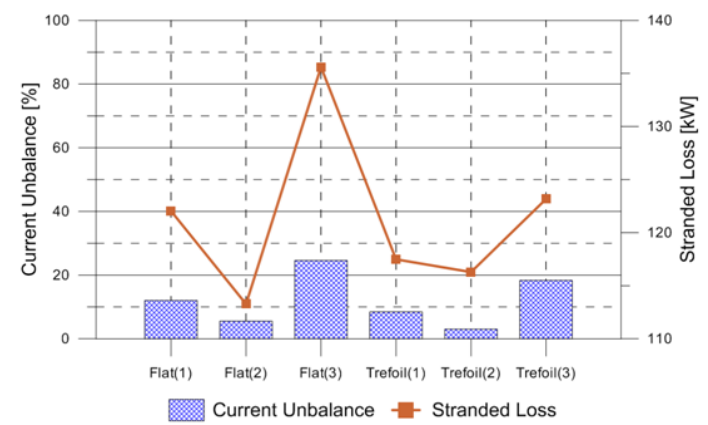

Fig. 10. Current unbalance and stranded loss of conductors 
The resistive loss of the iron tray is proportional to the magnetic flux density. The electric field $(E)$ is equal to the magnetic flux rate $(B)$ as noted in the Maxwell's equation in the differential form $\nabla \times E=-\partial B / \partial t$ [4].

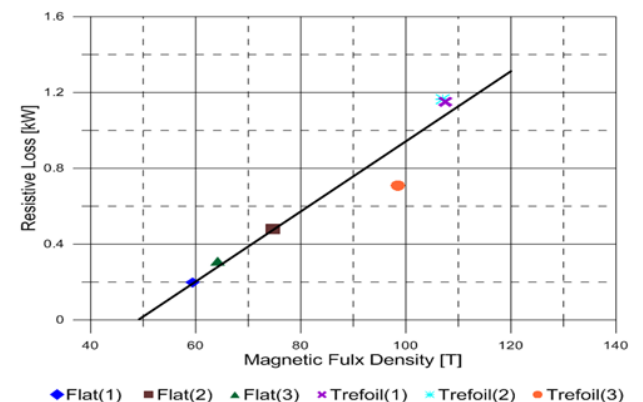

Fig. 11. Resistive losses versus magnetic flux density

Under sinusoidal flux conditions, core loss $\left(P_{v}\right)$ is computed in the frequency domain as the following [5].

$$
\begin{aligned}
& P_{v}=P_{h}+P_{c}+P_{e}=k_{h} f B_{m}^{2}+k_{c}\left(f B_{m}\right)^{2}+k_{e}\left(f B_{m}\right)^{1.5} \\
& \quad=K_{1} B_{m}^{2}+K_{2} B_{m}^{1.5} \\
& K_{1}=k_{h} f+k_{c} f^{2}, \quad K_{2}=k_{e} f^{1.5}
\end{aligned}
$$

Where

$B_{m} \quad$ : Maximum flux density

$k_{h}$ : Hysteresis core loss coefficient

$k_{c}$ : eddy-current core loss coefficient

$k_{e}$ : excess core loss coefficient

The eddy-current loss, which is often referred as classical loss, can be estimated with a constant value coefficient calculated as $k_{c}=\pi^{2} \sigma d^{2} / 6$ where $\sigma$ is the electrical conductivity and $d$ is the thickness of the material. For extracting the hysteresis and excess loss coefficients, a curve regression algorithm is used.

$$
f\left(K_{1}, K_{2}\right)=\sum_{i=1}^{n_{i}}\left[P_{v i}-\left(K_{1} B_{m i}^{2}+K_{2} B_{m i}^{1.5}\right)\right]^{2}=\min
$$

Parameters $B_{m i}$ and $P_{v i}$ are usually provided by the manufacturer. By minimizing the function the coefficient $K_{1}$ and $K_{2}$ are derived, then the hysteresis and excess loss coefficient can be obtained as $k_{h}=\left(K_{1}-k_{c} f_{0}^{2}\right) / f_{0}$, $k_{e}=K_{2} / f_{0}^{1.5}$ where $f_{0}$ is the testing frequency. In this case, the coefficients of the hysteresis, eddy-current, and excess core losses are defined a particular values as shown in Table 2 regarding to sheet iron material per meter cube.
Table 2. Core loss coefficients

\begin{tabular}{c|c|c|c}
\hline Section & $k_{c}$ & $k_{e}$ & $k_{h}$ \\
\hline Coefficient & 4.526 & 5.819 & 10.05 \\
\hline
\end{tabular}

The results of the finite element analysis show that the magnetic flux densities of the conductors and the current densities on the tray are distributed on the its surfaces.

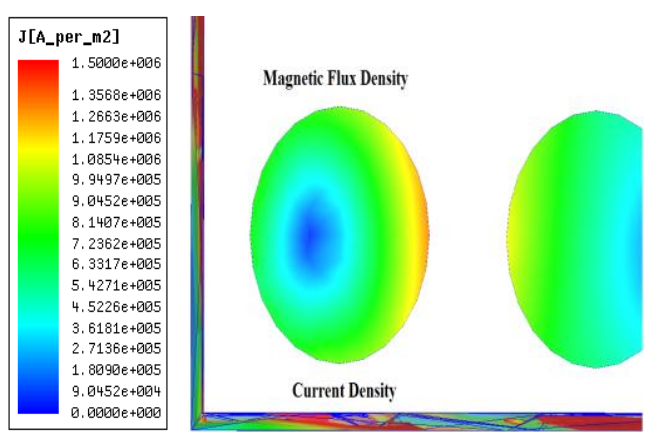

Fig. 12. Magnetic flux and current densities

The magnetic flux densities are described with contour lines at the front and bottom of the tray as shown in Fig 1314. If the Trefoil type is used for power cable arrangement, the magnetic flux density is higher than the Flat types under symmetry and asymmetry excitation current conditions.

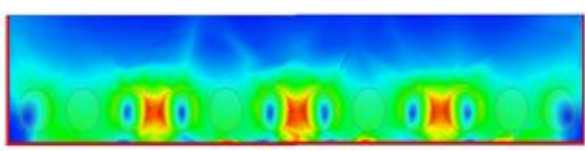

(a) Flat (1)
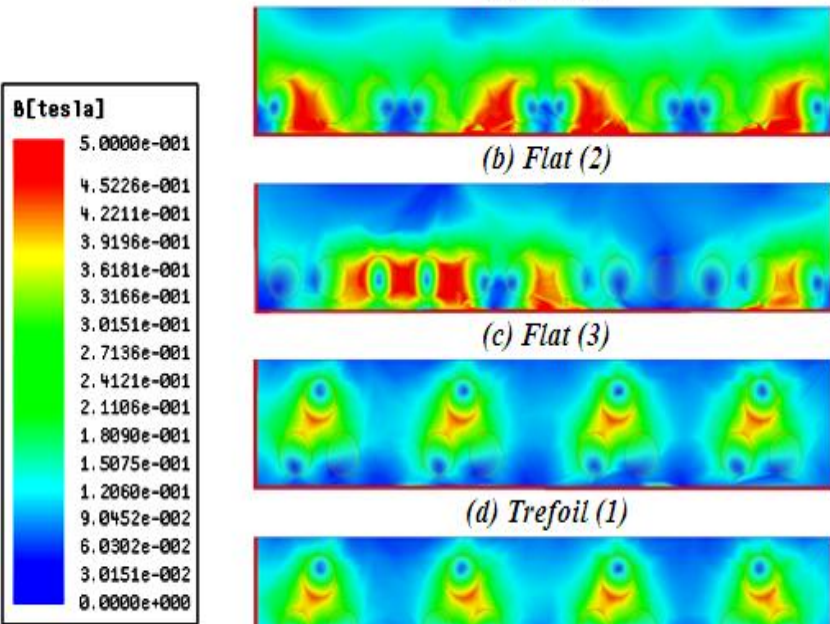

(b) Flat (2)

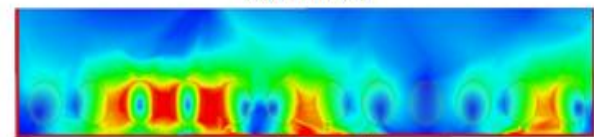

(c) Flat (3)

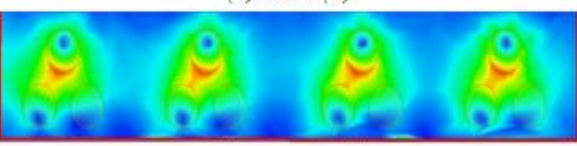

(d) Trefoil (1)

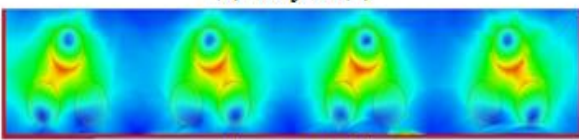

(e) Trefoil (2)

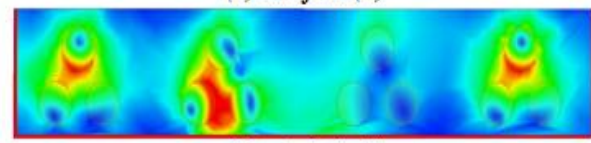

(f) Trefoil (3)

Fig. 13. Magnetic flux density at the front of the tray 


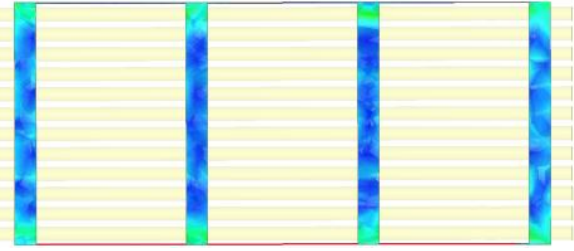

(a) Flat (1)

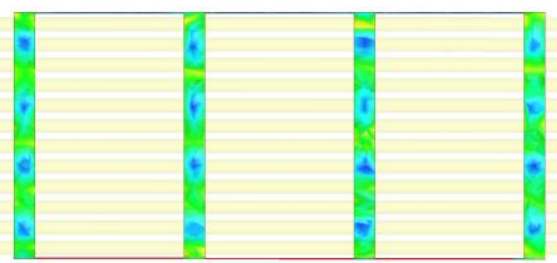

(b) Flat (2)

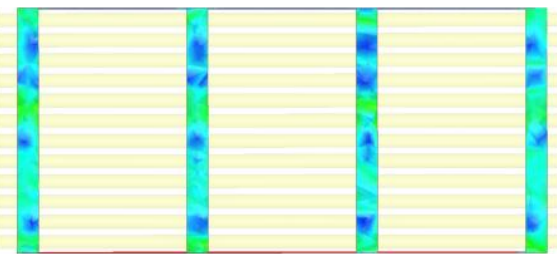

(c) Flat (3)

$4472 e+00$

1. $2663 e+061$

1. $0854 e+001$

$9.0452 e+000$

7. $2362 e+000$

3. $6181 e+000$

1. $8090 e+000$
$0.0000 e+000$

Flux density

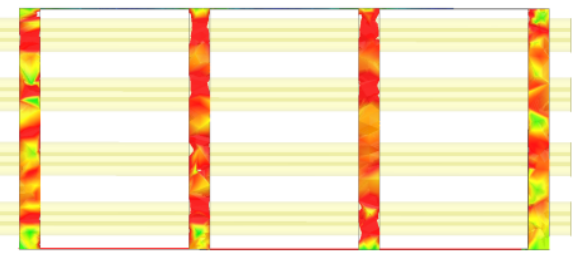

(d) Trefoil (1)

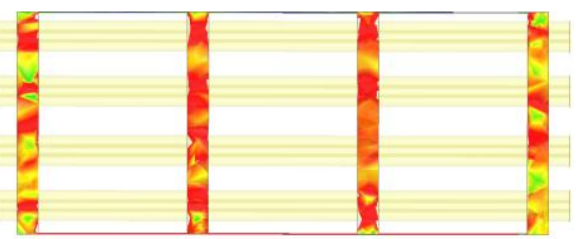

(e) Trefoil (2)

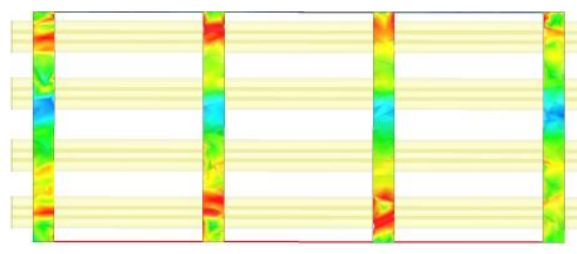

\section{(f) Trefoil (3)}

Trefoil

Fig. 14. Magnetic flux density on the bottom of tray

The equivalent elliptical loop (EEL) method is used for core loss calculations in time domain. In three dimensional finite element transient analysis, the scalar model of the equation (7) for soft materials is modified by equation (10)(13) with a typical value of hysteresis loss parameter $\beta=2$ and $C_{e}=8.76336[6]$.

$$
\begin{aligned}
& P_{h}(t)=\left\{\left|H_{x} \frac{d B_{x}}{d t}\right|^{\frac{2}{\beta}}+\left|H_{y} \frac{d B_{y}}{d t}\right|^{\frac{2}{\beta}}+\left|H_{z} \frac{d B_{z}}{d t}\right|^{\frac{2}{\beta}}\right\}^{\frac{\beta}{2}} \\
& P_{c}(t)=\frac{1}{2 \pi^{2}} k_{c}\left\{\left(\frac{d B_{x}}{d t}\right)^{2}+\left(\frac{d B_{y}}{d t}\right)^{2}+\left(\frac{d B_{z}}{d t}\right)^{2}\right\} \\
& P_{e}(t)=\frac{1}{C_{e}} k_{e}\left\{\left(\frac{d B_{x}}{d t}\right)^{2}+\left(\frac{d B_{y}}{d t}\right)^{2}+\left(\frac{d B_{z}}{d t}\right)^{2}\right\}^{0.75} \\
& C_{e}=(2 \pi)^{1.5} \cdot \frac{2}{\pi} \int_{0}^{\frac{\pi}{2}} \cos ^{1.5} \theta d \theta
\end{aligned}
$$

The three components of the core loss can be calculated with its own coefficient as shown in Fig. 15.

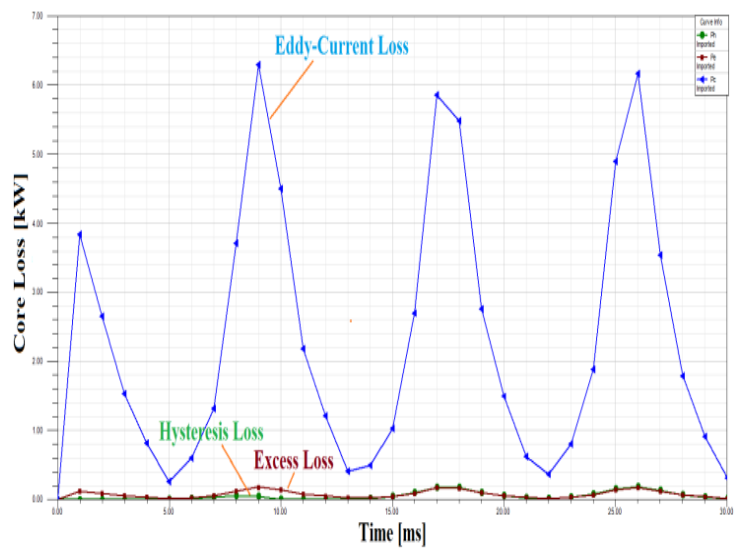

Fig. 15. Three components of the core loss

The simulation results show that the eddy-current, excess and hysteresis loss are occupied approximately 93\%, 5\%, and $2 \%$ respectively of core loss.

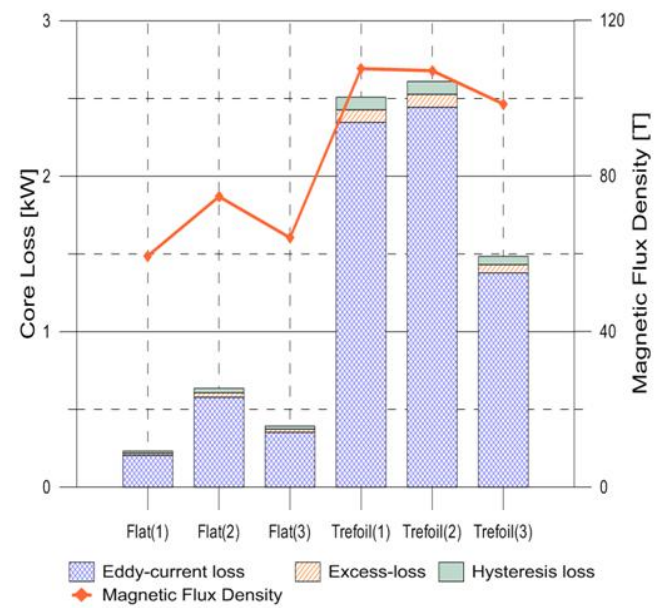

Fig. 16. Distribution of core loss in various cases 
The mean value of the magnetic flux density is reduced under excitation current with harmonics but the peak value of the core loss is very higher because of resultant flux densities at different relative phase angles of the third- and fifth harmonics.

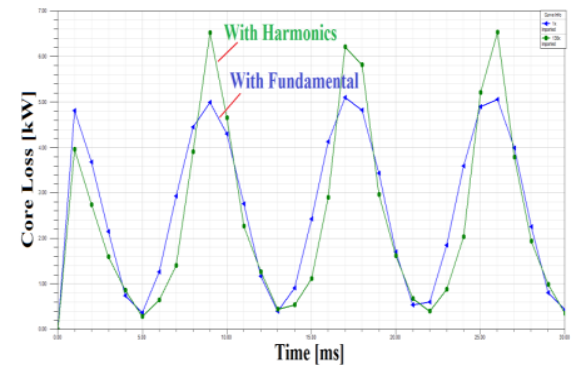

Fig. 17. Comparison of the peak value of core loss

The following figure shows the deviation between the peak values of core loss with/without harmonics. To reduce the peak value of the core loss associated with hot-spot temperature of tray, the Flat types are more effective than the others.

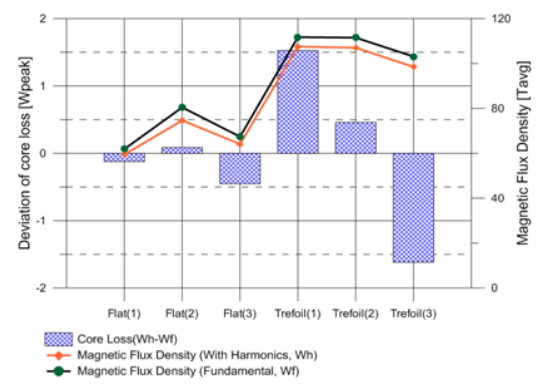

Fig. 18. Deviation between the peak values of core losses

The following illustration as a result of the curve fit in various measurements, the core loss is exponentially proportional to the magnetic flux density under $0.5 \mathrm{pu}$ and 1.0 pu excitation current as shown in Fig. 19.

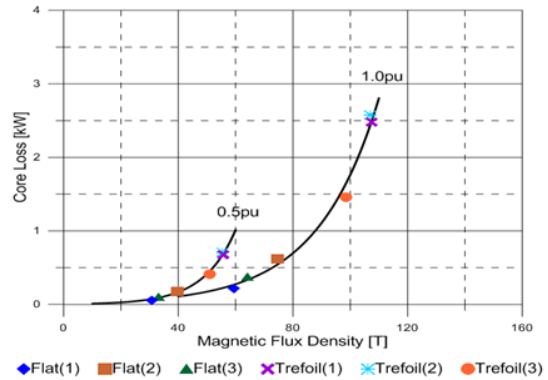

Fig. 19. Core loss versus Magnetic flux density

\section{Thermal-eddy current coupled analysis}

Temperature on the tray caused by the eddy-currents can be calculated refer to the equation (14).

$$
q=h_{c} A d T
$$

Where

$q$ : Heat transfer per unit time $(w)$

$h_{c}$ : Convective heat transfer coefficient $\left(W / m^{2} K\right)$

$A$ : Surface area for heat $\operatorname{transfer}\left(m^{2}\right)$

$d T$ : Temp difference between surface and fluid $(K)$

The property of the materials and simulation conditions are applied as shown Table 3. In this case, It is assumed that the radiation heat transfer emissivity coefficient of copper and iron is applied 0.78, 0.95 respectively and the convective heat transfer coefficient is 25 for free convection of air cooling. Ambient temperature surrounding the power cable trays installed in the closed structure applied 25 degrees Celsius.

Table 3. Conditions of coupled analysis

\begin{tabular}{l|c|c|c}
\hline \multirow{2}{*}{ Sections } & \multicolumn{3}{c}{ Property of the materials } \\
\cline { 2 - 4 } & Cooper & Iron & $\mathrm{Al}$ \\
\hline $\begin{array}{l}\text { Thermal Conductivity } \\
\left(W / m^{2} \mathrm{~K}\right)\end{array}$ & 385 & 79.5 & 205 \\
\hline $\begin{array}{l}\text { Radiation heat transfer } \\
\text { emissivity coefficient }\end{array}$ & 0.78 & $0.87 \sim 0.95$ & 0.2 \\
\hline $\begin{array}{l}\text { Convective heat transfer } \\
\text { coefficient }\left(\mathrm{W} / \mathrm{m}^{2} \mathrm{~K}\right)\end{array}$ & \multicolumn{3}{|c}{$5 \sim 25$} \\
\hline $\begin{array}{l}\text { Ambient } \\
\text { temperature }\left({ }^{\circ} \mathrm{C}\right)\end{array}$ & \multicolumn{3}{|c}{25} \\
\hline
\end{tabular}

The results of thermal-eddy current coupled analysis indicate that the hot-spot temperature in a variety of the power cable arrangements is proportional to the magnetic flux density.

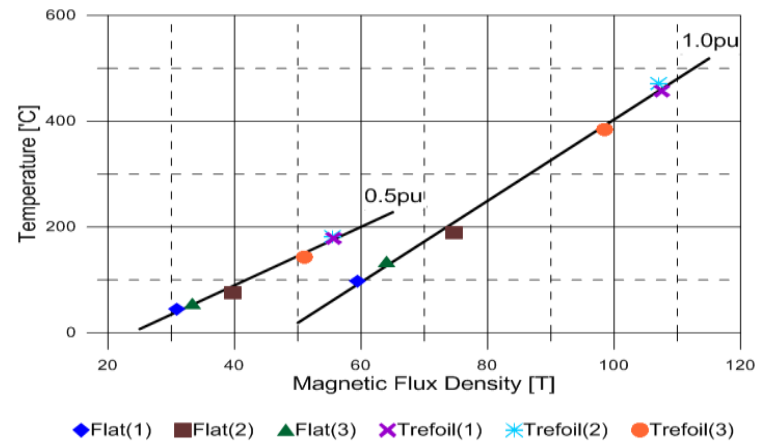

Fig. 20. Hot-spot temperature versus magnetic flux density

In order to secure the maximum transmission power to the load, both the Flat (2) and Trefoil (2) types are beneficial compare to the other types. Where a large amount of current being supplied to the load, the use of non-magnetic materials such as aluminum steel is required to eliminate the hot-spot temperature of the tray. 


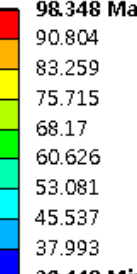

30.448 Min
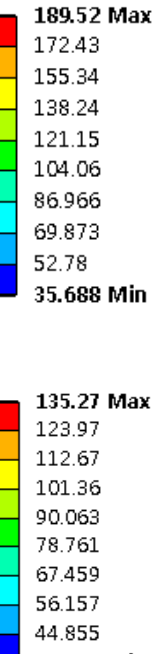

33.554 Min
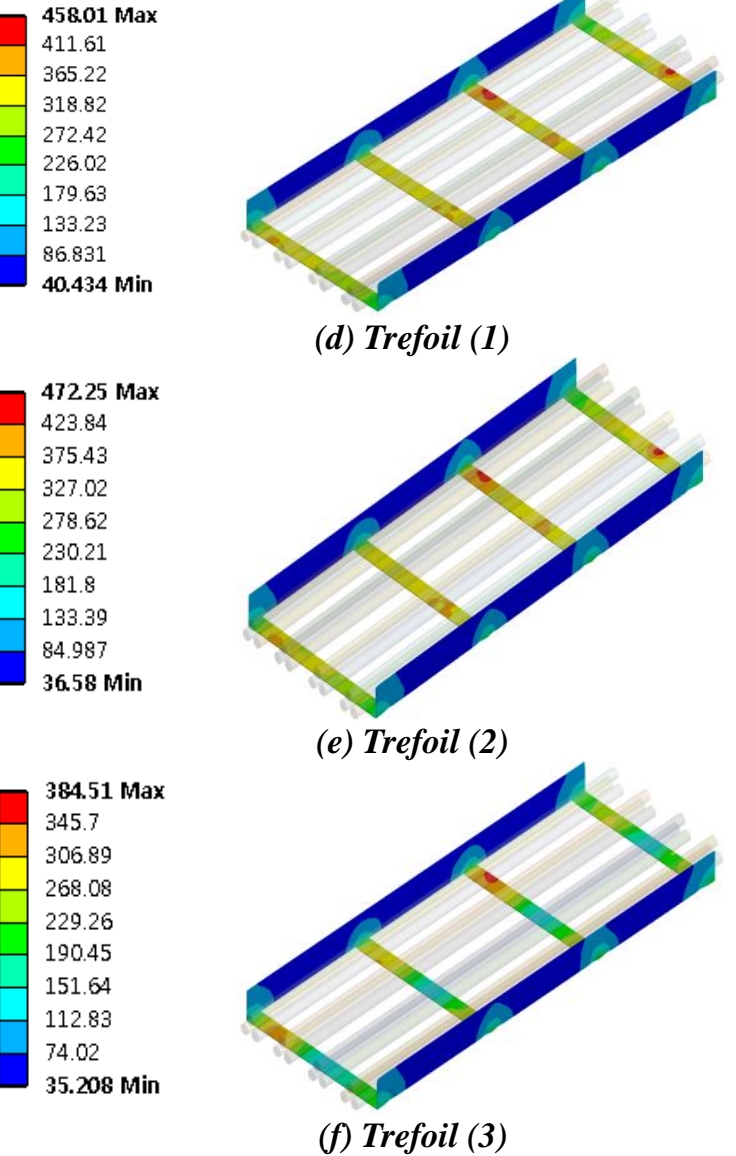

Fig 21. Hot-spot temperature of magnetic materials

\section{Conclusion}

An excessively high temperature deterioration of the power cable insulation is directly related to excessively high temperatures. To analyze the nonspecific such as overheating problems on the cable tray, electromagnetic finite element analysis under a variety of cable arrangements has been conducted by eddy-current and thermal coupled solver of the Maxwell and Ansys Mechanical Software. Its simulation results show main factors causing the problems and practical method available for power cable arrangements through analyzes of the effects of harmonics, the impact of asymmetric flux, decomposition of three components in the core loss and current distribution of the conductors. In particular, the analysis of core loss components is important factors to ensure the performance and to select the appropriate materials of all electrical equipment. The coefficient of eddy-current, hysteresis and excess loss can be calculated easily from core loss curve provided by manufacturer or experimental data.

The hot spot temperature has been significantly reduced by maintaining symmetry magnetic flux on the basis of the analysis results. This electromagnetic finite element method and thermal-eddy current coupled analysis would be helpful not only to solve the similar problems caused by electromagnetic phenomena such as overheating of the metal conduit and isolated phase buses of the generator but also to maximize the transmit power considering high order harmonics in the field.

\section{References}

[1] Y. Du, Y, Z, Yuan, and X, H, Wang "Current Distribution in Parallel Single-Core Cables on Metal Tray", IEEE Transactions on industry applications, vol 44. No. 6, November, December, 2008, pp.1886-1891.

[2] Z. H. Yuan and Y. Du "harmonic Impedance of Single-Core Armored Cables", IEEE 0-7803-8110-6/03, 2003, pp.45-48.

[3] Schneider, Electric, "Electrical installation guide according to IEC international standards", 2010.

[4] Zoya Popovic, Branko D. Provic, "Introductory Electromagnetics", Prenticel Hall, pp. 359-377.

[5] D. Lin, P. Zhou, W. N. Fu, Z. Badics, and Z. J. Cendes, "A dynamic core loss model for soft ferromagnetic and power ferrite materials in transient finite element analysis", IEEE Trans. On Mag., vol. 40, no. 2, March 2004.

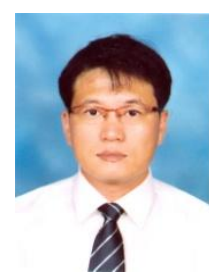

Hyung-Joo Choi received M.S. degree in electrical engineering from the ChungNam national university and $\mathrm{He}$ has worked as a manager in charge of expert service team in the power utility company, KOMIPO in Korea. His research interests are optimization and stability of power systems and electric machines. 
Heung-Ho Lee received Ph.D. degree in electrical engineering from Seoul national university and $\mathrm{He}$ has served as a professor in the ChungNam national university. His research interests are computer science for power system and electric machines. 